\title{
Trihedral lattice towers geometry optimization
}

\author{
Leysan Akhtyamova, Anton Chepurnenko*, Marina Rozen, and Ebrahim Al-Wali \\ Don State Technical University, 344002, Rostov-on-Don, Russia
}

\begin{abstract}
The problem of trihedral lattice towers geometry optimization, the width of which varies linearly with height, has been considered. The variable parameters were the support width at the base and top point, as well as the cross-sectional areas of the lattice and chords. A restriction was introduced on the mass constancy. The objective function was the potential strain energy, the maximum displacement and the first frequency of natural vibrations. In the first and second cases the optimum corresponds to the minimum of the objective function, and in the third - to the maximum. The solution was performed by the finite element method in combination with four nonlinear optimization methods: the interior point method, surrogate optimization method, genetic algorithm and pattern search method. The efficiency of each of the listed optimization methods has been compared by the authors.
\end{abstract}

\section{Introduction}

Currently, in the practice of building tower structures, lattice structures are mainly used in the form of spatial trusses of three- and tetrahedral, in some cases, polyhedral sections. The most rational of these are the triangular lattice structures, the advantages of which are the lowest metal consumption, due to the absence of the need to use a stiffness diaphragm in their design, as well as the least flexibility to uneven foundation settlement [1].

To date, the main recommendations for the creation of the lattice tower structures' rational structural forms have been developed as well as the methods for their calculation. A large number of the original tower-type structures have been built. However, some topical aspects have not been fully investigated; they include, in particular, the optimization of the triangular lattice tower structures' geometric parameters. There are some publications on these structures' cross-sectional shape optimization [2,3], however, the issues of finding the optimal relationship between the geometric parameters of the structure as a whole have not been properly reflected in the literature.

The proposed optimization technique on the example of trihedral lattice tower, the width of which varies linearly in height (Fig. 1), has been considered. The cross-section of the chords $A_{\mathrm{p}}$ and the lattice $A_{\mathrm{r}}$ does not change in height. The height of the tower is constant and equal to $40 \mathrm{~m}$, the number of panels is 10 . The values $A_{\mathrm{p}}$ and $A_{\mathrm{r}}$, as well as the tower width at the base $B_{0}$ and at the top point $B_{1}$, are used as variable parameters. Nonlinear constraint is introduced - the tower mass, which is a non-linear function of $B_{0}, B_{1}, A_{\mathrm{p}}$ and $A_{\mathrm{r}}$, must be constant. For simplicity, the load here and below is represented by a

*Corresponding author: anton_chepurnenk@mail.ru 
concentrated unit force $F$ at the upper end, lying in a horizontal plane, the direction of which is determined by the angle $\alpha$. Such a load can simulate a top-mounted wind turbine. At the lower points, the tower is hinged fixed. The restrictions are introduced on the minimum and maximum values of $B_{0}, B_{1}, A_{\mathrm{p}}$ and $A_{\mathrm{r}}$.

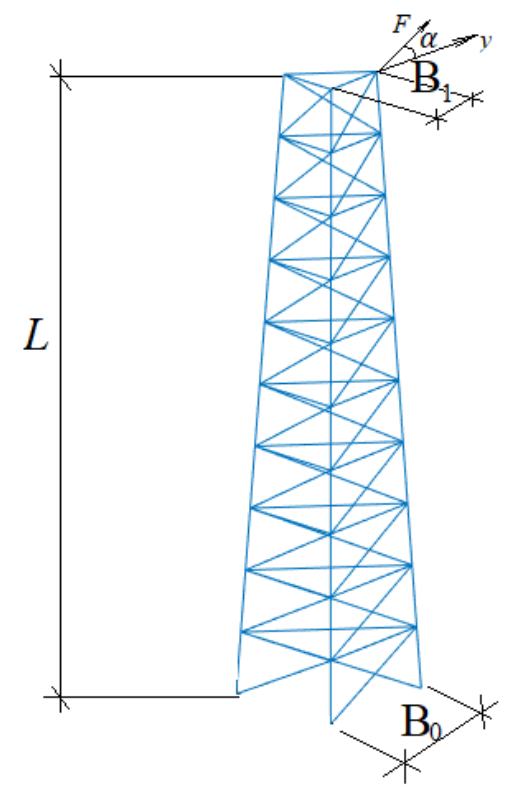

Fig. 1. The tower calculation scheme

\section{Methods}

At the first stage, the problem with the following initial data: $\alpha=0$, at the initial search point $B_{0}=2 \mathrm{~m}, B_{1}=1 \mathrm{~m}, A_{\mathrm{p}}=5 \cdot 10^{-3} \mathrm{~m}^{2}, A_{\mathrm{r}}=0.1 \mathrm{~A}_{\mathrm{p}}$ was solved. The lower and upper limits for the parameters $B_{0}, B_{1}, A_{\mathrm{p}}$ and $A_{r}$ are presented in Table 1 .

Table 1. Lower $(l b)$ and upper $(u b)$ limits for variable parameters.

\begin{tabular}{|c|c|c|c|c|}
\hline parameter & $B_{0}, \mathrm{~m}$ & $B_{1}, \mathrm{~m}$ & $A_{\mathrm{p}}, \mathrm{m}^{2}$ & $A_{\mathrm{r}}, \mathrm{m}^{2}$ \\
\hline$l b$ & 0.5 & 0.5 & 0.001 & 0.0001 \\
\hline$u b$ & 4 & 3 & 0.1 & 0.1 \\
\hline
\end{tabular}

The optimization problem in the Matlab environment using the finite element method in combination with nonlinear optimization methods included in the Optimization Toolbox and Global Optimization Toolbox packages has been solved. Potential strain energy (PSE), maximum displacement of the structure and natural vibration frequency were selected as the objective function.

The choice of the potential strain energy as the objective function is explained by the fact that it is an integral measure determining the stress-strain state level [4]. The smaller the value of $W$, the better the system resists external influences [5].

The choice of the maximum displacement of the structure as an objective function does not require justification. And the first natural frequency was chosen as an optimization criterion, since it characterizes the structure's ability to resist the mechanical resonance occurrence. The higher the first frequency, the more difficult it is to cause resonance in it. 
The problem of nonlinear optimization was considered as the problem of finding the minimum (for potential strain energy or deflection) or maximum (for the first natural frequency) of the nonlinear objective function of multiple variables $f(x)$ with the restrictions:

$$
\left\{\begin{array}{c}
c(x) \leq 0 \\
c e q(x)=0 \\
A \cdot x \leq b \\
\text { Aeq } x=b e q \\
l b \leq x \leq u b
\end{array}\right.
$$

Here $x$ is the vector containing variable parameters, $A$ and $A_{\text {eq }}$ are the matrices, $c(x)$ and $c e q(x)$ are the nonlinear functions of several variables that return a scalar, $l b$ and $u b$ are the elower and upper bounds for variable variables, respectively.

Trihedral lattice supports were modelled using spatial two-node finite elements of rod with three degrees of freedom at the node (Fig. 2).

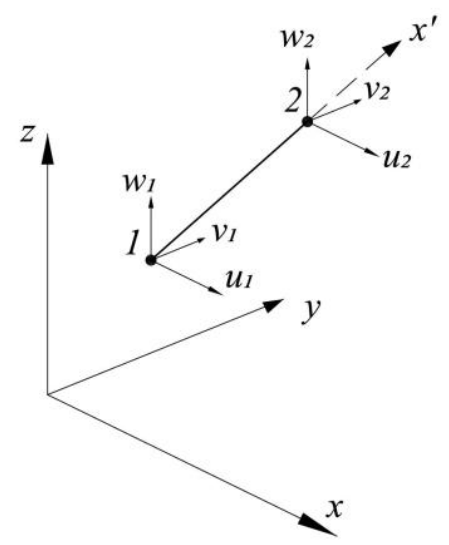

Fig. 2. Rod finite element for calculation taking into account three degrees of freedom in a node

The potential strain energy was determined by the formula:

$$
W=\frac{1}{2}\{U\}^{T}[K]\{U\},
$$

where $[K]$ is a structural stiffness matrix, $\{U\}$ is the nodal displacements vector determined from the solution of the system of equations of the FEM:

$$
[K]\{U\}=\{F\},
$$

where $\{F\}$ is a vector of nodal loads.

The local stiffness matrix of an element working only in tension-compression is determined by the well-known formula:

$$
\left[K^{e}\right]=\frac{E A}{l}\left[\begin{array}{cc}
1 & -1 \\
-1 & 1
\end{array}\right],
$$

where $E$ is the modulus of the material elasticity, $A$ is the cross-sectional area of the element, $l$ is the finite element length.

The natural frequencies $\omega$ were determined from the equation: 


$$
\left|[K]-\omega^{2}[M]\right|=0,
$$

where $[M]$ is a diagonal mass matrix.

For the initial point of the search, the values of the potential strain energy $W$, the maximum displacement $v$ and the first frequency of natural vibrations were $W=8 \mathrm{~J}, v=$ $1.6 \cdot 10^{-2} \mathrm{~m}, \omega=7.06 \mathrm{~s}^{-1}$.

To solve the nonlinear optimization problem, the following methods were used:

1. Interior point method [6]. This method performs optimization using the gradient information of the objective function In the Matlab environment interior point method is implemented in the fmincon function of the Optimization Toolbox package.

2. Surrogate optimization method [7]. The essence of this method is to replace the objective function with its approximation (surrogate) and search for a minimum for the surrogate. The approximation of the objective function in this method is based on randomly generated points. The Global Optimization Toolbox implements this method in the surrogateopt function.

3. Pattern search [8]. This method forms a set of points around the current point, called a mesh, and searches for a point at which the value is lower than at the current point. The mesh is formed by adding the coordinates of the current point with the product of a set of vectors, called a pattern, by a scalar, called the size of the mesh. If the algorithm finds a point at which the value of the objective function is better than the current one, it becomes the current one at the next step. The set of vectors (pattern) can be fixed or randomly generated. The Global Optimization Toolbox implements this method in the patternsearch function.

4. Genetic algorithm. This algorithm, in contrast to the classical approaches, creates a set of points at each iteration. The best point in the population approaches the optimal solution. This algorithm uses four main types of rules at each stage to create the next generation from the current population: inheritance, selection, crossing and mutation, mimicking natural selection in nature. A detailed description of the algorithm and examples of its use in problems of optimization of building structures are presented in $[9,10]$. The Global Optimization Toolbox implements this method in the ga function.

\section{Results and Discussion}

The optimal values of the varied parameters obtained on the basis of three optimization criteria and the corresponding values of the objective function when using the interior point method are presented in Table 2. The criterion of minimum PSE and minimum displacement in this case give the same results.

Table 2. Optimal values of the varied parameters and the corresponding values of the objective function when using the interior point method

\begin{tabular}{|c|c|c|c|c|c|c|c|}
\hline criterion & $\begin{array}{c}B_{0}, \\
{[\mathrm{~m}]}\end{array}$ & $\begin{array}{c}B_{1}, \\
{[\mathrm{~m}]}\end{array}$ & $A_{p},\left[\mathrm{~m}^{2}\right]$ & $A_{r},\left[\mathrm{~m}^{2}\right]$ & $W,[\mathrm{~J}]$ & $v,[\mathrm{~m}]$ & $\omega,\left[\mathrm{s}^{-1}\right]$ \\
\hline $\begin{array}{c}\text { minimum } \\
\text { PSE, } \\
\text { minimum } v\end{array}$ & 4 & 3 & 0.0046 & 0.0005 & 2 & $4.1 \cdot 10^{-3}$ & 13.05 \\
\hline maximum $\omega$ & 4 & 2.67 & 0.0045 & 0.0006 & 2.1 & $4.2 \cdot 10^{-3}$ & 13.14 \\
\hline
\end{tabular}

It can be seen from the presented table that the potential strain energy and the greatest displacement of the structure reach their minimum at the upper boundaries of the parameters $B_{0}$ and $B_{1}$. 
The surrogate optimization method and the genetic algorithm in this problem showed a much worse result (Table 3 and Table 4). It should be noted that there is no explicit stopping criterion in the surrogate optimization method, and, as a rule, the number of iterations is limited. This number was taken equal to 2000 .

Table 3. Optimal values of the varied parameters and the corresponding values of the objective function when using the surrogate optimization method

\begin{tabular}{|c|c|c|c|c|c|c|c|}
\hline criterion & $B_{0},[\mathrm{~m}]$ & $\begin{array}{c}B_{1}, \\
{[\mathrm{~m}]}\end{array}$ & $A_{p},\left[\mathrm{~m}^{2}\right]$ & $A_{r},\left[\mathrm{~m}^{2}\right]$ & $W,[\mathrm{~J}]$ & $v,[\mathrm{~m}]$ & $\begin{array}{c}\omega,\left[\mathrm{s}^{-}\right. \\
1]\end{array}$ \\
\hline $\begin{array}{c}\text { minimum } \\
\text { PSE }\end{array}$ & 3.57 & 2.68 & 0.0042 & 0.0007 & 2.6 & $5.1 \cdot 10^{-3}$ & 11.97 \\
\hline minimum $v$ & 3.48 & 2.67 & 0.005 & 0.0003 & 2.6 & $5.2 \cdot 10^{-3}$ & 11.44 \\
\hline $\begin{array}{c}\text { maximum } \\
\omega\end{array}$ & 3.7 & 2.86 & 0.0042 & 0.0007 & 2.4 & $4.8 \cdot 10^{-3}$ & 12.32 \\
\hline
\end{tabular}

Table 4. Optimal values of the varied parameters and the corresponding values of the objective function when using the genetic algorithm

\begin{tabular}{|c|c|c|c|c|c|c|c|}
\hline criterion & $B_{0},[\mathrm{~m}]$ & $\begin{array}{c}B_{1}, \\
{[\mathrm{~m}]}\end{array}$ & $A_{p},\left[\mathrm{~m}^{2}\right]$ & $A_{r},\left[\mathrm{~m}^{2}\right]$ & $W,[\mathrm{~J}]$ & $v,[\mathrm{~m}]$ & $\omega,\left[\mathrm{s}^{-1}\right]$ \\
\hline $\begin{array}{c}\text { minimum } \\
\text { PSE }\end{array}$ & 2.45 & 1.03 & 0.0034 & 0.0016 & 7.9 & $1.57 \cdot 10^{-2}$ & 7.37 \\
\hline $\begin{array}{c}\text { minimum } \\
v\end{array}$ & 2.23 & 1.19 & 0.0048 & 0.0006 & 6.5 & $1.3 \cdot 10^{-2}$ & 7.83 \\
\hline $\begin{array}{c}\text { maximum } \\
\omega\end{array}$ & 3.36 & 2.58 & 0.003 & 0.0014 & 3.8 & $7.5 \cdot 10^{-3}$ & 10.02 \\
\hline
\end{tabular}

It can be seen from Table 4 that for this problem the genetic algorithm showed an unsatisfactory result. The values of the objective functions obtained by this method are better than at the starting point, but not significantly.

The pattern search method shows the results, which are closest to the interior point method (Table 5).

Table 5. Optimal values of the varied parameters and the corresponding values of the objective function when using the pattern search method

\begin{tabular}{|c|c|c|c|c|c|c|c|}
\hline criterion & $B_{0},[\mathrm{~m}]$ & $\begin{array}{c}B_{1}, \\
{[\mathrm{~m}]}\end{array}$ & $A_{p},\left[\mathrm{~m}^{2}\right]$ & $A_{r},\left[\mathrm{~m}^{2}\right]$ & $W,[\mathrm{~J}]$ & $v,[\mathrm{~m}]$ & $\begin{array}{c}\omega,\left[\mathrm{s}^{-}\right. \\
1]\end{array}$ \\
\hline $\begin{array}{c}\text { minimum } \\
\text { PSE }\end{array}$ & 4 & 2.92 & 0.0046 & 0.005 & 2.1 & $4.1 \cdot 10^{-3}$ & 13.07 \\
\hline minimum $v$ & 3.93 & 2.94 & 0.0046 & 0.005 & 2.1 & $4.2 \cdot 10^{-3}$ & 12.9 \\
\hline $\begin{array}{c}\text { maximum } \\
\omega\end{array}$ & 4 & 2.87 & 0.0046 & 0.005 & 2.1 & $4.1 \cdot 10^{-3}$ & 13.07 \\
\hline
\end{tabular}

Here, as in the case of using the internal point method, the minimum of displacement and potential strain energy are achieved near the upper boundary of the parameters $B_{0}$ and $B_{1}$. Further, we varied the upper limits $u b$ of these parameters, and it was found that, regardless of the $u b$ values, the optimal deflection and PSE values are always achieved when $B_{0}$ and $B_{1}$ are maximum. Thus, when optimizing the towers with a width linearly varying in height, it is sufficient to choose the areas of chords and lattices or the ratio $A_{\mathrm{p}} / A_{\mathrm{r}}$ as variable parameters during optimization.

It was also found that varying the angle $\alpha$, which determines the unit force direction, does not significantly affect the optimal parameters $B_{0}, B_{1}, A_{\mathrm{p}}, A_{\mathrm{r}}$. 


\section{Conclusion}

The optimization problem for a triangular lattice tower, the width of which varies linearly with height, has been solved. In the solution, the interior point method, surrogate optimization method, genetic algorithm and pattern search method were used. It was found that the interior point method is the most effective for the considered problem. Using the criteria of minimum potential strain energy and minimum displacement leads to the same results. It was found that the minimum of displacement and potential strain energy are achieved at the upper boundaries of the parameters $B_{0}$ and $B_{1}$, which makes it possible to exclude these dimensions from the number of varied parameters.

\section{References}

1. I. Badertdinov et al., IOP Conference Series: Materials Science and Engineering 412, 012005 (2018).

2. L. Sabitov, I. Kuznetsov, I. Badertdinov, Power of Tatarstan 1, 47-51 (2016).

3. A. Chepurnenko et al., IOP Conference Series: Materials Science and Engineering 1083, 012012 (2021).

4. G. Vasil'kov, Evolutionary tasks of structural mechanics: a synergetic paradigm (InfoServis, Rostov-on-Don, 2003).

5. G. Vasil'kov, Theory of adaptive evolution of mechanical systems (Terra-Print, Rostovon-Don, 2007).

6. R. Byrd, M. Hribar, J. Nocedal, SIAM Journal on Optimization 9, 877-900 (1999).

7. H. Gutmann, Journal of Global Optimization 19, 201-227 (2001).

8. T. Kolda, R. Lewis, V. Torczon, A generating set direct search augmented Lagrangian algorithm for optimization with a combination of general and linear constraints. (Technical Report SAND2006-5315, Sandia National Laboratories, 2006).

9. I. Serpik, A. Alekseytsev, P. Balabin, Periodica polytechnica civil engineering 61, 471482 (2017).

10. I. Serpik, I. Mironenko, V. Averchenkov, Procedia Engineering 150, 1311-1316 (2016). 